\title{
Cryo-injury Induced Heart Regeneration in the Axolotl and Echocardiography and Unbiased Quantitative Histology to Evaluate Regenerative Progression
}

\author{
Anita Dittrich ${ }^{1}$, Henrik Lauridsen ${ }^{1}$ \\ ${ }^{1}$ Department of Clinical Medicine (Comparative Medicine Lab), Aarhus University
}

\section{Corresponding Author}

Henrik Lauridsen

henrik@clin.au.dk

\section{Citation}

Dittrich, A., Lauridsen, H. Cryo-injury

Induced Heart Regeneration in the

Axolotl and Echocardiography and

Unbiased Quantitative Histology to

Evaluate Regenerative Progression.

J. Vis. Exp. (171), e61966,

doi:10.3791/61966 (2021).

\section{Date Published}

May 10, 2021

DOI

$10.3791 / 61966$

\section{Abstract}

The urodele amphibians, salamanders and newts, represent the phylogenetic group closest to mammals capable of performing complete myocardial regeneration following ventricular resection. The resection model has generated a basic knowledge of the processes involved in cardiac repair. However, the model does not relate well to clinical situations in which tissue damage, apoptosis, necrosis, fibrosis, and hypertrophy are all key detrimental consequences of ischemia-induced myocardial infarctions rather than tissue removal. On the other hand, cryoinjury-induced myocardial infarction resembles ischemia-induced myocardial infarction more closely. Here we provide a detailed description of the cryoinjury procedure in the axolotl (Ambystoma mexicanum), which provides a tool for investigating basic mechanisms in cardiac repair in a tetrapod model. Additionally, we provide quantitative methods for estimating infarction size non-invasively in vivo with echocardiography and for measuring infarction size precisely with unbiased quantitative histology ex vivo.

URL

jove.com/video/61966

\section{Introduction}

Ischemic heart disease is a leading cause of fatalities globally $^{1,2}$. Ischemia induced myocardial infarction results in widespread cell death in cardiomyocytes ${ }^{3}$. Although rapid medical intervention can reduce the risk of immediate demise, the ensuing fibrotic response that humans share with traditional mammalian model animals (mouse, rat, rabbit, pig, etc.) results in scar tissue formation that can ultimately lead to cardiac hypertrophy, arrhythmias, and heart failure ${ }^{4}$. Contrary to mammals, cardiac regeneration is well established in some non-amnionic animal models such as zebrafish ${ }^{5}$ and salamanders ${ }^{6}$. Traditionally, cardiac regeneration has been studied in these species after partial ventricular resection or pinching $5,6,7,8,9,10$. However, in 2011, three groups independently developed a cryoinjuryinduced myocardial infarction technique in zebrafish ${ }^{11,12,13}$. The cryoinjury technique results in necrosis and apoptosis 
in a major part of the zebrafish heart ventricle and an initial accumulation of fibrotic tissue that more closely models the pathological development of the mammalian heart following ischemic infarction compared to ventricular resection ${ }^{11,12,13}$. Additionally, methodological comparisons of cryoinjury-induced myocardial infarction to ischemiainduced myocardial infarction by coronary artery ligation in the mouse and the pig have proven the cryoinjury technique to be a useful alternative in mammalian animal models ${ }^{14,15}$. Inspired by the methods involved in the zebrafish cryoinjury model $^{16,17}$ we have developed a similar model in the axolotl $^{18}$, an amphibian renowned for its regenerative capabilities $^{19}$, that allows investigation of the mechanisms involved in cardiac repair in this tetrapod after tissue damage rather than tissue removal.

Here we present a detailed protocol on how to perform cryoinjury induced myocardial infarction in the axolotl. We place special emphasis on rapid and minimally invasive crucial steps that increase survival, recovery, and experimental reproducibility. Additionally, we provide instructions for appropriate techniques for evaluating anatomical regeneration in vivo using echocardiography and ex vivo using unbiased stereology based quantitative histology.

Cryoinjury-induced myocardial infarction in the axolotl can be applied to investigate basic mechanisms involved in myocardial regeneration in this tetrapod. The axolotl is tolerant of cryoinjury-induced myocardial infarction, which affects at least $45 \%$ of the ventricle, resulting in a decrease in stroke volume and cardiac output without any behavioral changes in its relatively languid lifestyle, however, more severe injuries will potentially lead to decreased animal health.
In short, to induce cryoinjury, a ventral incision is made on the thorax of an anaesthetized axolotl. The ventricle is accessed using blunt dissection and a precooled cryoprobe is applied for $10 \mathrm{~s}$ to induce cryoinjury. The ventral incision is sutured and the animal quickly recovers with no signs of behavioral changes after consciousness is regained. Control/ sham animals receive similar treatment but experience a noncooled cryoprobe. Cardiac performance can be monitored using echocardiography (high frequency ultrasound systems necessary, $\geq 20 \mathrm{MHz}$ ) as exhaustively described on healthy axolotl hearts previously ${ }^{20}$, and infarction fraction can be estimated non-invasively and repeatedly during the regenerative process. Infarcted hearts can be harvested at any time during the 3-month regenerative process, cryosectioned for histology, and stained using standard procedures (e.g., eosin \& hematoxylin or Masson's trichrome staining). In particular, Masson's trichrome staining allows a clear distinction between infarcted and healthy parts of the ventricle. The infarction fraction of the ventricle is determined using stereological techniques previously described for cardiac studies $^{21}$.

\section{Protocol}

This protocol complies to all institutional (Aarhus University) and national (Danish National Animal Experiments Inspectorate protocol\# 2015-15-0201-00615) animal care regulations and guidelines.

\section{Preparation of cryoprobe}

1. Cut three strands of copper wire with $1 \mathrm{~mm}$ diameter into $10 \mathrm{~cm}$ pieces.

2. Coil copper strands into one string. 
3. With a gas torch melt one end of the copper string to form a spherical ending with a $2 \mathrm{~mm}$ diameter.

NOTE: Copper has a high thermal conductivity $(401 \mathrm{~W}$ $\mathrm{m}^{-1} \mathrm{~K}^{-1}$ at $0^{\circ} \mathrm{C}$ ) and can be molded at easily manageable temperatures (melting point $1084.6{ }^{\circ} \mathrm{C}$ ) but a copper surface has a tendency to corrode over time especially if it comes into contact with blood, which lowers thermal conductivity markedly. Thus, a copper based cryoprobe must be polished occasionally. Alternative more stable metals that can also be used for cryoprobes are silver (thermal conductivity $428 \mathrm{~W} \mathrm{~m}^{-1} \mathrm{~K}^{-1}$ at $0^{\circ} \mathrm{C}$, melting point $961.8^{\circ} \mathrm{C}$ ) and gold (thermal conductivity $318 \mathrm{~W} \mathrm{~m}^{-1} \mathrm{~K}^{-1}$ at $0{ }^{\circ} \mathrm{C}$, melting point $1064.2^{\circ} \mathrm{C}$ ).

4. Remove the piston from a 2-mL syringe and pull off the soft rubber head.

5. Insert the non-spherical end of the copper string through the syringe and tie it to the pistol

6. Put the piston back in place in the syringe (Figure 1A). NOTE: When the cryoprobe is not in use, the copper string should be protected by drawing the piston fully back. When in use, the piston is pushed all the way in to expose the copper string to the cooling bath.

\section{Cryoinjury induced myocardial infarction}

1. Anaesthetize axolotl by immersing it in $200 \mathrm{mg} / \mathrm{L}$ of benzocaine anesthetic. Thirty minutes of immersion will yield $\sim 2.5 \mathrm{~h}$ of complete anesthesia.

NOTE: Benzocaine is insoluble in water and should first be solubilized in $3 \mathrm{~mL}$ (per $200 \mathrm{mg}$ benzocaine) acetone that is subsequently added to the water bath. Prolonged immersion in benzocaine can impair recovery, therefore time in benzocaine should be monitored carefully.
2. Place the axolotl with the ventral side up on a surgery dish (head to the left for a right-handed surgeon). Immobilize by encasing all body parts except the thorax in tissue wipes soaked in anesthetic solution (Figure 1B). NOTE: Encasing the animal in wet tissue wipes ensures a moist environment crucial for an aquatic amphibian and eliminates the need for vet ointment on eyes. Use autoclaved instruments in subsequent steps to ensure sterility during surgery.

3. Use iridectomy scissors to make a $\sim 1-\mathrm{cm}$ longitudinal incision in the skin slightly right to the midline of the thorax (Figure 1C).

4. Use forceps to free the cartilage plates of the pectoral girdle.

NOTE: The pectoral girdle is a delicate structure and care should be taken not to damage its elements as this can impair later wound healing and animal locomotion and welfare.

5. Gently open the exposed pericardium with an incision without damaging underlying cardiac structures. Before pericardial fluid leaks out aspirate this into a $1 \mathrm{~mL}$ syringe carrying a blunt 2 Gauge needle (Figure 1D).

6. Place blunt forceps with a set opening $(\sim 1 \mathrm{~cm})$ in the wound to keep wound edges and cartilage plates apart (Figure 1E).

7. Place the cryoprobe in liquid $\mathrm{N}_{2}$ with the copper wire exposed for at least 2 minutes.

NOTE: If the mass of the copper wire is too small (i.e., it is too short) or if it is cooled insufficiently, an undesirable incomplete transmural cryoinjury can result.

8. Gently wipe the exposed ventricle with a cotton swab or firmly rolled tissue wipes to make sure no fluid is present on the ventricle surface (Figure 1F). 
9. Remove the cryoprobe cooled to $-196{ }^{\circ} \mathrm{C}$ from the liquid $\mathrm{N}_{2}$ and apply it directly to the exposed ventricle (Figure 1H). It will attach to the ventricle. Cryoprobe placement should be on the lateral side of the ventricle toward the apex to avoid damage to other cardiac structures and allow for subsequent transversal sections for microscopy containing both healthy and infarcted myocardium. After $10 \mathrm{~s}$ apply the previously collected pericardial fluid to the cryoprobe tip (Figure 1I). This will thaw the probe and allow for detachment from the ventricle.

NOTE: Applying the cryoprobe on a ventricle with excessive water results in either incomplete cryoinfarction, if the probe is heated too rapidly, or too extensive myocardial damage, if a thin water layer covering the full surface of the ventricle is instantaneously frozen. Neither situation is desirable. In the case of a successful induction of cryoinjury, a small red circular swelling will appear on the ventricle (Figure 1J). When handling sham animals, a non-cooled cryoprobe is placed in a similar fashion for $10 \mathrm{~s}$ (Figure 1G).

10. Place the pericardium on top of the ventricle, remove the forceps that kept the wound open, and rearrange the cartilage plates of the pectoral girdle.

NOTE: If performing subsequent echocardiography to evaluate cardiac function, it is crucial that all air pockets, even minute bubbles that are prone to occur under the cartilage plates, are removed as these induce imaging artifacts. These bubbles can be removed by gently pressing the tissue or by adding autoclaved amphibian Ringer's solution $(6.6 \mathrm{~g} / \mathrm{L} \mathrm{NaCl}, 0.15 \mathrm{~g} / \mathrm{L} \mathrm{KCl}, 0.15 \mathrm{~g} / \mathrm{L}$ $\mathrm{CaCl}_{2}, 0.2 \mathrm{~g} / \mathrm{L} \mathrm{NaHCO}_{3}$ in distilled water) to the wound surface to dissolve the air bubbles.
11. Suture the skin (3-5 stiches) using dissolvable thread (6-0) (Figure 1K).

12. Cover the surgery area with wet tissue wipes lifted above the wound and then soak with anesthetic solution.

13. Place the surgery dish with the animal on ice for $2 \mathrm{~h}$ to allow the initial formation of a wound epidermis, sealing the wound edges before the animal is exposed to its nonsterile aquatic environment.

14. Transfer the animal to a clean container with aquarium water. The animal it should be regaining consciousness by this time. Observe the animal until it has regained full consciousness and coordinated movements. Cease feeding and water changing for two days post-surgery and keep the axolotl alone in its own aquarium to allow wound closure before disturbances are reintroduced.

NOTE: After regaining consciousness, most axolotls have a desire to gulp air. To ease this process and to limit the amount of movement needed, it can be beneficial to lower the water level in the aquarium shortly after surgery for 1-2 days post-surgery. A low concentration of benzocaine $(20 \mathrm{mg} / \mathrm{L})$ can be used as analgesic for 24 h post-surgery.

\section{Non-invasive measurement of infarction fraction with echocardiography}

1. Anesthetize axolotl as described in step 2.1.

2. Place the animal in a supine position in a small container with 3-5 mm of anesthesia solution covering the ventral surface.

3. Place the ultrasound transducer along the long axis of the animal slightly to the right of the midline of the thoracic region. 
4. For a two-dimensional estimation of infarction fraction [IF(ultrasound, 2D)], translate the transducer until the infarction zone and ventricle appear maximal on B-mode (Figure 2A-D, Supplementary Material 1-2).

5. Acquire $\geq 3$ cardiac cycles with $>50$ frames/s.

6. Measure the cross-sectional area of the ventricle (CSAv) and of the infarction zone (CSAi) at end-diastole and estimate the two-dimensional infarction fraction by assuming a spherical ventricle shape and hemispherical infarction zone shape using the equation:

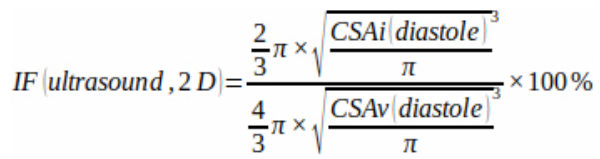

NOTE: To evaluate the geometrical assumptions of the shape of the ventricle and infraction zone, the transducer can be rotated $90^{\circ}$ to display the short axis view of the heart (Figure 2E-F, Supplementary Material 3).

7. For a three-dimensional estimation of the infarction fraction, position the transducer to either the left or right until only a small portion of the ventricle appears in view at the end of ventricular diastole.

8. In steps ( $\mathrm{z}$ ) of $0.5 \mathrm{~mm}$ (for small animals, body mass $\leq$ $20 \mathrm{~g}$ ) or $1 \mathrm{~mm}$ (large animals, body mass $>20 \mathrm{~g}$ ) move the transducer across the ventricle and acquire at least 3 cardiac cycles per step/slice.

NOTE: For reasonable precision 8 - 12 slices $(n)$ should be acquired.

9. Measure CSAv and CSAi at end-diastole for each slice (j) and estimate three-dimensional infarction fraction [IF(ultrasound, 3D)] without any geometrical assumptions of ventricle and infraction shape using the equation:
IF (ultrasound , $3 D=\frac{Z \times \sum_{j=1}^{n} \operatorname{CSAi}(\text { diastole })_{j}}{Z \times \sum_{j=1}^{n} \operatorname{CSAv}(\text { diastole })_{j}} \times 100 \%$

NOTE: Both two-dimensional and three-dimensional acquisition can be used to measure cardiac function (stroke volume and cardiac output) geometrically as described in sections 3 and 6 of Dittrich et al. $(2018)^{20}$. Likewise, pulsed wave velocity-acquired velocity time integrals (Figure 2G) can also be used to measure cardiac function as described in section 3 in Dittrich et al. $(2018)^{20}$.

10. Extract regions of interest of drawn CSAv and CSAi in step 3.9 as binary masks with $z$ spacing between slices to three-dimensionally model the ventricle and infarction zone at end diastole and end systole (Figure

\section{H, Supplementary Material 4).}

\section{Unbiased quantitative histology to measure infarction fraction}

1. Explore the heart of an anesthetized axolotl described in step $2.2-2.6$.

2. Using a 1-mL syringe equipped with a 26 -gauge needle, slowly inject $5 \mu \mathrm{L} / \mathrm{g}$ body mass of $1000 \mathrm{IU} / \mathrm{mL}$ heparinized amphibian Ringer's solution into the sinus venosus and wait $2 \mathrm{~min}$ to let the anticoagulant distribute in the circulation.

3. Euthanize by decapitation and brain pithing and excise the entire heart by first cutting the inflow vessels and then the outflow tract.

4. Transfer the heart by gently lifting it by the outflow tract to a $60 \times 15 \mathrm{~mm}^{2}$ Petri dish containing amphibian Ringer's solution. 
5. Under magnification, wash out any remaining blood in the heart by gently filling the sinus venosus with $100 \mathrm{IU} / \mathrm{mL}$ of heparinized Ringer's solution.

NOTE: While pre-fixation of the heart can be conducted by perfusing the heart with buffered formalin or paraformaldehyde, this is not necessary, since sectioning is carried out at a freezing temperature. Subsequently, fixation of the sections on glass slides can be performed. A fully washed heart will appear pale and whitish and contain no traces of red blood cells. Small blood clots on the surface of ventricle and atria are likely to form. They do not affect precision in the subsequent quantitative histology procedure.

6. Transfer the heart to lint-free tissue paper to blot for 1 $\min$.

7. Prepare a cryomold of adequate size by applying a single drop of optimal cutting temperature compound and transfer the heart to the drop. Squeeze the heart gently to the bottom.

8. Apply more optimal cutting temperature compound to fill up the cryomold and make sure that the ventricle stays at the bottom. Leave the mold for 5 min to let the optimal cutting temperature compound distribute in the sample.

9. Snap freeze the mold by placing it on a lid from a pipette tip box floating on liquid nitrogen.

NOTE: For a more controlled snap freezing, hearts can be stored for 1 h-overnight in 10-30\% sucrose solution and snap freezing can be performed using liquid nitrogen-cooled isopentane (2-methylbutane). In our experience, the end result is not markedly different.

10. Temper the cryomold in a cryostat set at -18 to $-22{ }^{\circ} \mathrm{C}$ for at least $1 \mathrm{~h}$ before sectioning.
11. Section sample at $10 \mu \mathrm{m}$ slice thickness and collect every $10^{\text {th }}$ slice to provide a slab thickness $(t)$ of $100 \mu \mathrm{m}$. Continue sectioning until entire heart is sectioned.

NOTE: Different slice and slab thicknesses can be used, however it is important that slice collection starts at the very point the blade touches the ventricle and that the entire heart is sectioned with at least $10-12$ evenly spaced slabs. More than one slice per slab can be collected to provide duplicate sets.

12. Stain tissue sections with standard protocols for hematoxylin \& eosin or Masson's Trichrome to provide a clear distinction between epicardium, myocardium, valves, and infarction zone.

13. Digitize the tissues section using a camera microscope or slide scanner at sufficient magnification to clearly reveal the trabeculated structure of the axolotl ventricle (Figure 21).

14. Load the image stack into ImageJ (https://imagej.nih.gov/ $\mathrm{ij} /$ index.html) and initialize the cell counter plugin (https:// imagej.nih.gov/ij/plugins/cell-counter.html).

15. Run a multipurpose grid macro (https://imagej.nih.gov/ij/ macros/Multipurpose_grid.txt) and generate a point grid with an adequate grid density.

NOTE: Grid density (grid area) depends on the sample size and morphology. To provide a desirable precision, at least 100-200 intersections of grid points and each of the structures of interest should be ensured.

16. Count the number of intersections between point grid and structures of interest using the cell counter plugin (Figure 2I, magnification on the right).

NOTE: It is important for precision to include only those intersections that appear precisely at the aim point of the crosshair. 
17. Calculate the infarction fraction from the pre-defined point grid area $[\mathrm{Ai}(\mathrm{p})$ and $\operatorname{Av}(\mathrm{p})]$, the slab thickness, and the number of point grid intersections at each slab $\left(P_{j}\right)$ with infarcted myocardium and total ventricular myocardium using the formula:

$$
I F(\text { stereology })=\frac{A i(p) \times t \times \sum_{j=1}^{n} P_{j}}{A v(p) \times t \times \sum_{j=1}^{n} P_{j}} \times 100 \%
$$

\section{Representative Results}

In axolotls with a body mass of $11.8 \pm 1.3 \mathrm{~g}$ and a total length of $11.8 \pm 0.5 \mathrm{~cm}$, the cryoinjury procedure performed with a 2$\mathrm{mm}$ (diameter) cryoprobe results in an infarcted area covering $45.4 \pm 14.2 \%$ of the ventricular myocardium at 7 days post injury in which the infarction zone is fully developed [Figure 2I, compare section from healthy heart pre infraction (top) and 7 days post infraction (bottom)]. The procedure has a mortality rate of $2.2 \%$ (2/90 animals). The procedure results in a welldefined injury zone that can be visualized, quantified, and modeled with non-invasive echocardiography (Figure 2CH, Supplementary material 1-4). Initially, cryoinjury induced myocardial infarction significantly effects cardiac function, reducing the stroke volume to $62.2 \%$ and the cardiac output to $73.9 \%$ at 7 days post injury relative to pre injury with a gradual recovery of form and function over three months (data not shown). No behavioral changes following myocardial cryoinjury are observed in the relatively tranquil axolotl. 


\section{jove}
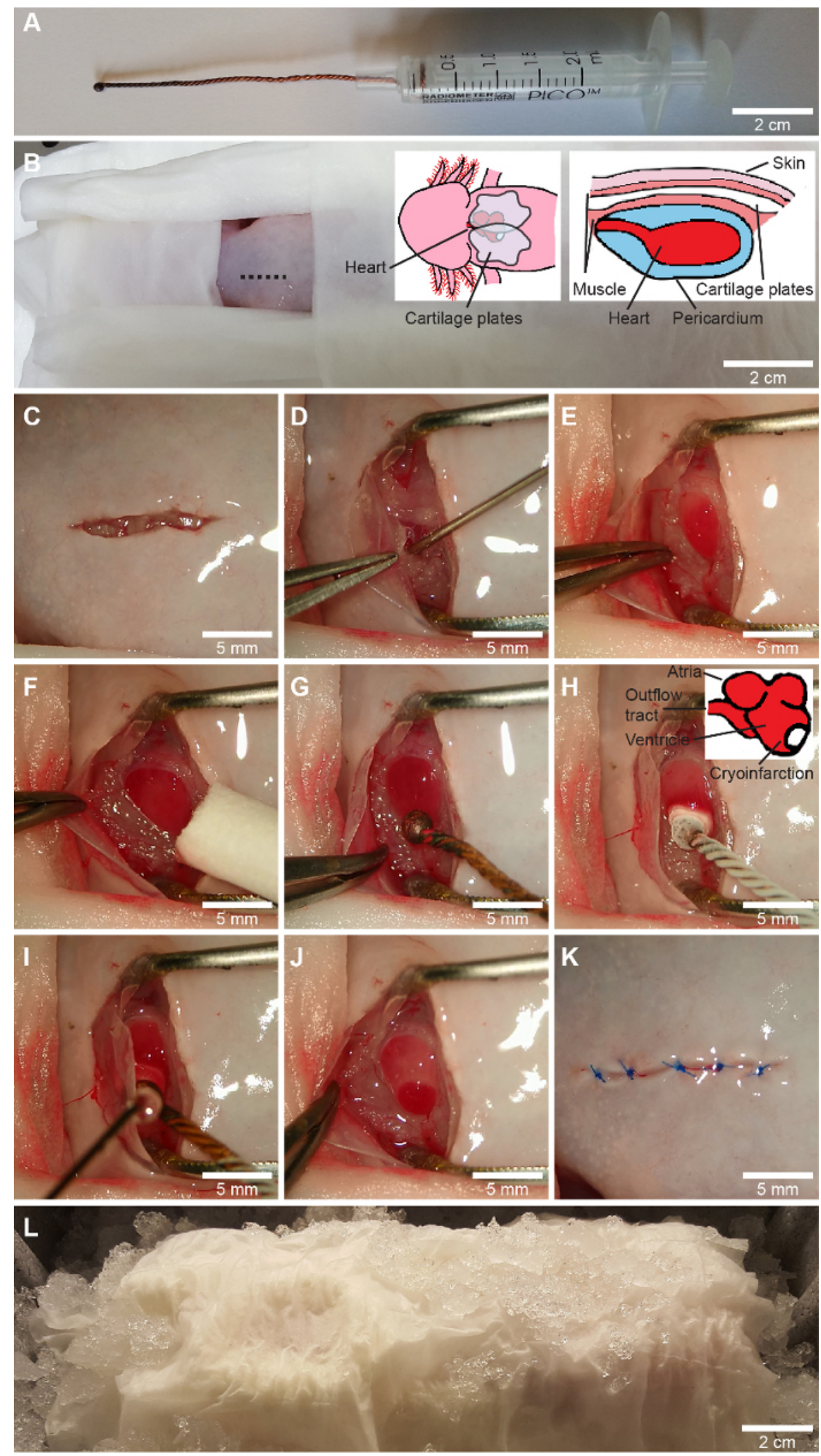
Figure 1: Cryoinfarction procedure. A, a custom made cryoprobe is made by coiling three copper wires, melting the tip into a sphere and attaching the wire to the piston of a $2.5-\mathrm{mL}$ syringe. B-L, the cryoinfarction procedure. Following the encasing of all body parts except the surgery zone in wet tissue wipes (B), a ventral incision on the thorax slightly to the right of the midline (shown on $\mathbf{B}$ ) is performed with iridectomy scissors $(\mathbf{C})$, and the pectoral girdle is freed by stump dissection. The pericardium is gently opened with an incision and the pericardial fluid is aspirated into a 1-mL syringe using a blunt 23-gauge needle (D). The ventricle is exposed and the wound is kept open with forceps with a predefined opening width (E) and any remaining fluid on the ventricle is wiped off $(\mathbf{F})$. For sham surgery a non-cooled cryoprobe $(\mathbf{G})$ is applied in the same way as for cryoinjury, in which the cryoprobe cooled in liquid nitrogen $\left(-196{ }^{\circ} \mathrm{C}\right)$ is applied to the lateral wall of the ventricle toward the apex $(\mathbf{H})$. After $10 \mathrm{~s}$, the pericardial fluid is reapplied to the cryoprobe to release it from the ventricle (I). This yields a clearly defined cryoinjury zone $(\mathbf{J})$. Finally, the pericardium and the pectoral girdle are laid down on top of the heart and the skin is sutured (3-5 stiches) with a dissolvable suture (K). The animal is left on ice for 2 hours to initiate the wound healing process $(\mathbf{L})$. Cranial is toward the left on $\mathbf{B}-\mathbf{L}$, representing the orientation of the animal for a surgeon with a dominant right hand. Please click here to view a larger version of this figure. 

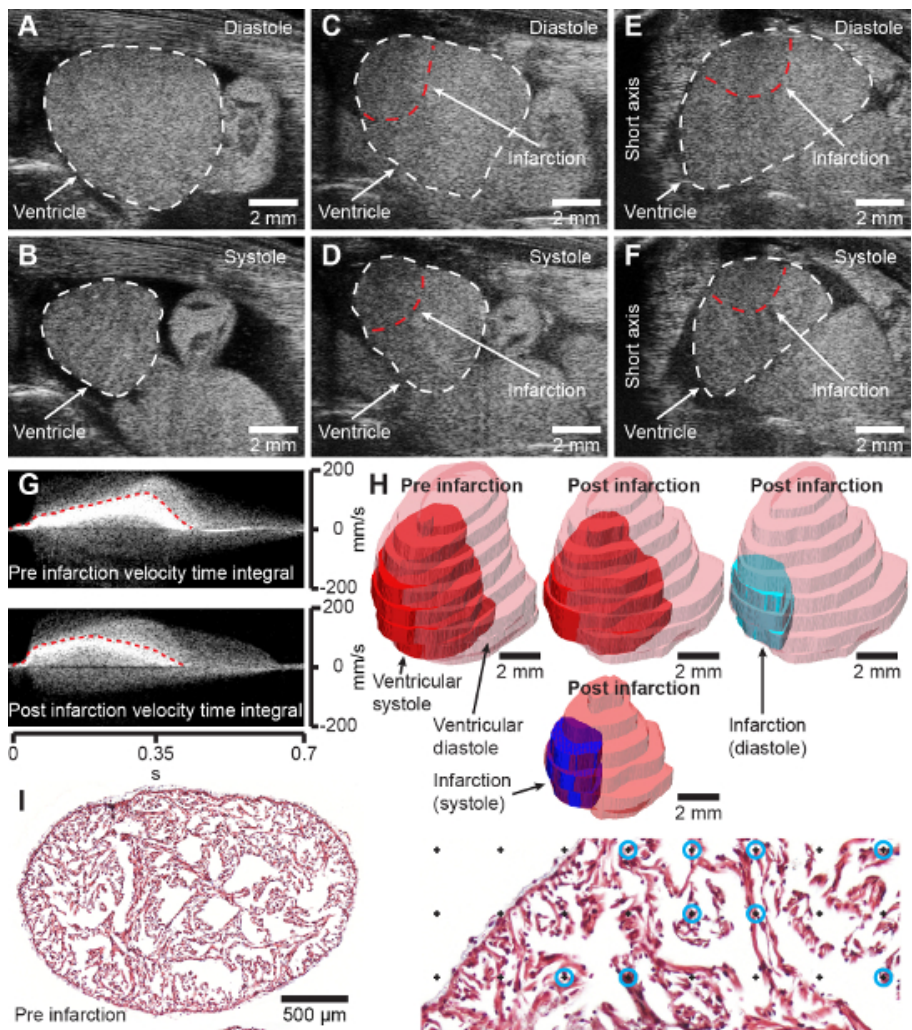

Ventricular
diastole

Infarction

(systole) $\rightarrow \longrightarrow$
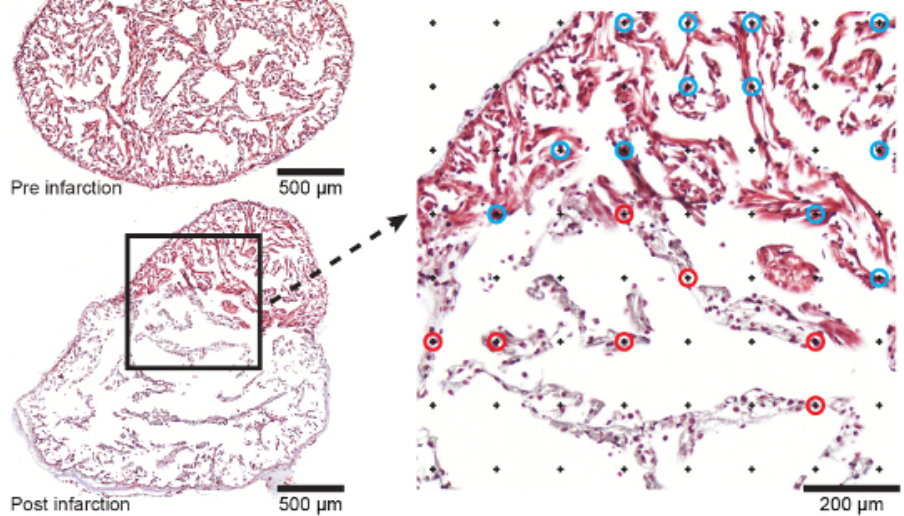

Figure 2: Anticipated results. A and B, B-mode long axis images of healthy axolotl ventricle in diastole (A) and systole (B). C and D, B-mode long axis images of cryoinjured (2 days post injury) axolotl ventricle in diastole (C) and systole (D). E and F, B-mode short axis images of cryoinjured (2 days post injury) axolotl ventricle in diastole (E) and systole (F). G, Pulse wave Doppler acquired velocity time integrals of pre (top) and 2-day post infarcted heart (bottom). H, Modeling of pre infarction and 2-day post infarcted heart from three-dimensional ultrasound acquisition. I, representative transversal histology sections through the axolotl ventricle 4 days post sham (top) and 7 days post infarction (bottom) stained with Masson's Trichrome. Magnification on right shows point grid for stereological measurement of infarction fraction. Points marked by blue and red circles represent intersection with healthy (blue circles) and infarcted (red circles) tissue. Cranial is toward the right in A-D and animals right is toward the left in E-F, which represents the conventional display of echocardiographic images. Please click here to view a larger version of this figure. 
Supplementary material 1: Long axis, healthy ventricle pre infarction, B-mode (see Figure 2A-B). Please click here to download this video.

Supplementary material 2: Long axis, infarcted ventricle (2 days post infarction), B-mode (see Figure 2C-D). Please click here to download this video.

Supplementary material 3: Short axis axis, infarcted ventricle (2 days post infarction), B-mode (see Figure 2E-F). Please click here to download this video.

Supplementary material 4: Three-dimensional interactive models of the same heart as seen in Figure $\mathbf{2 A}-\mathbf{H}$ and Supplementary material 1-3 pre- and post-infarction. Launch the interactive PDF file in Adobe Acrobat Reader 9 or higher. Click the model to activate the 3D feature. Rotate, zoom, and pan the model using the cursor. In the model tree to the left side of the screen, all segments can be activated/deactivated or made transparent. The model tree is constructed as a hierarchy that contains several sub-layers that can be opened (by selecting +). Please click here to download this file.

\section{Discussion}

To minimize experimental variation, the surgical procedure of the cryoinjury should follow sterile procedures and surgical training should be conducted on a number of animals before attempting to use animals for specific regenerative experiments. With training, the cryoinjury procedure can be conducted on a large range of axolotl sizes and ages, from juveniles $(5 \mathrm{~g}, 7 \mathrm{~cm})$ to large adults $(100 \mathrm{~g}, 25 \mathrm{~cm})$. It is critical that the cryoprobe has a sufficient size and is cooled adequately to provide a robust and repeatable cryoinjury to the axolotl ventricle. For very small animals $(5-8 \mathrm{~g}, 7-9 \mathrm{~cm})$, the cryoprobe can be constructed with a smaller diameter. While it is imperative that the cryoinjury protocol is conducted using an anesthetic with analgesic properties like benzocaine and MS-222 (or with the additional application of a secondary analgesic agent), the follow up echocardiography can be conducted with other anesthetics with only limited analgesic properties, such as propofol, which has been described to affect cardiac function less than benzocaine and MS-222 in the axolotl ${ }^{22}$.

The cryoinjury procedure is limited in the sense that it does not produce an ischemia-induced myocardial infarction by coronary artery occlusion in the same manner as coronary artery ligation procedures that more closely resemble clinical cases of myocardial infarction in humans. However, the ligation-based method is not applicable in the trabeculated amphibian heart with very little coronary vasculature and a mostly luminal oxygen supply. Also, cryoinjury-induced myocardial infarction has been described to recapitulate most of the pathological consequences of ischemia-induced myocardial infarctions ${ }^{14,16}$. The injury zone generated by cryoinjury is highly localized to the tissue in proximity to the cryoprobe and, though this does not resemble a complex and branchlike infarction resulting from coronary blockage, it is advantageous in an experimental setting as the border zone between healthy and infarcted tissue can easily be recognized, and the progression of newly formed cardiomyocytes can be studied.

The axolotl is a considerably larger animal than the zebrafish with a more complicated cardiovascular system, including a heart that consists of three chambers (two atria, one ventricle) and a functional, though not anatomical, separation of blood flow in oxygenated and deoxygenated currents ${ }^{23}$ compared to the two chambered heart and serial flow system found in teleosts. The cryoinjury procedure previously described in the zebrafish does not involve post-operative suturing of the 
incised ventral surface ${ }^{16,17}$. This is necessary in the axolotl to avoid unnecessary exposure of the heart to the non-sterile aquatic environment in the animal's laboratory habitat.

Unbiased stereology-based quantitative histology is currently underreported in the regenerative field in which most quantitative measurements of infarction fraction relies on area drawing on histological section at the mid infarction zone and in some cases two neighboring sections ${ }^{24}$. Since the concept of stereology can be applied in histological examinations in any model species to provide more robust and unbiased measurements, we propose that this freely available method should be incorporated in quantitative evaluations of heart regeneration not only in the axolotl, but in all regenerative species.

\section{Disclosures}

The authors have nothing to disclose.

\section{Acknowledgments}

We wish to acknowledge Casper Bindzus Foldager, Asger Andersen, and Michael Pedersen (all at the Department of Clinical Medicine, Aarhus University) and David Gardiner (Department of Developmental and Cell Biology, University of California, Irvine) for help in the initial development of the axolotl cryoinjury model and ultrasound examination.

\section{References}

1. Forouzanfar, M.H. et al. Assessing the global burden of ischemic heart disease, part 2: analytic methods and estimates of the global epidemiology of ischemic heart disease in 2010. Global Heart. 7, 331-342 (2012).

2. Go, A.S. et al. Executive summary: heart disease and stroke statistics--2014 update: a report from the
American Heart Association. Circulation. 129, 399-410 (2014).

3. Murry, C.E., Reinecke, H.,Pabon, L.M. Regeneration gaps: observations on stem cells and cardiac repair. Journal of the American College of Cardiology. 47, 1777-85 (2006).

4. Laflamme, M. A., Murry, C.E. Heart regeneration. Nature. 473, 326-335 (2011).

5. Poss, K. D., Wilson, L. G., Keating, M. T. Heart regeneration in zebrafish. Science. 298, 2188-2190 (2002).

6. Oberpriller, J. O., Oberpriller, J. C. Response of the adult newt ventricle to injury. Journal of Experimental Zoology. 187, 249-260 (1974).

7. Cano-Martínez, A. et al. Functional and structural regeneration in the axolotl heart (Ambystoma mexicanum) after partial ventricular amputation. Archivos de Cardiología de México. 80, 79-86 (2010).

8. Witman, N., Murtuza, B., Davis, B., Arner, A., Morrison, J. I. Recapitulation of developmental cardiogenesis governs the morphological and functional regeneration of adult newt hearts following injury. Developmental Biology. 354, 67-76 (2011).

9. Mercer, S. E., Odelberg, S. J., Simon, H-G. A dynamic spatiotemporal extracellular matrix facilitates epicardialmediated vertebrate heart regeneration. Developmental Biology. 382, 457-469 (2013).

10. Piatkowski, T., Mühlfeld, C., Borchardt, T., Braun, T. Reconstruction of the myocardium in regenerating newt hearts is preceded by transient deposition of extracellular matrix components. Stem Cells and Development. 22, 1921-1931 (2013). 
11. Chablais, F., Veit, J., Rainer, G., Jaźwińska. The zebrafish heart regenerates after cryoinjury-induced myocardial infarction. BMC Developmental Biology. 11 (21), (2011).

12. González-Rosa, J. M., Martín, V., Peralta, M., Torres, M., Mercader, N. Extensive scar formation and regression during heart regeneration after cryoinjury in zebrafish. Development. 138, 1663-74 (2011).

13. Schabel, K., Wu, C. C., Kurth, T., Weidinger, G. Regeneration of cryoinjury induced necrotic heart lesions in zebrafish is associated with epicardial activation and cardiomyocyte proliferation. PLoS ONE. 6 (4), e18503 (2011).

14. Van den Bos, E. J., Mees, B. M. E., de Waard, M. C., de Crom, R., Duncker, D. J. A novel model of cryoinjury-induced myocardial infarction in the mouse: a comparison with coronary artery ligation. The American Journal of Physiology: Heart and Circulatory Physiology. 289, H1291-H1300 (2005).

15. Yang, Y. et al. Characterization of cryoinjury-induced infarction with manganese-and gadolinium-enhanced MRI and optical spectroscopy in pig hearts. Magnetic Resonance Imaging. 28, 753-766 (2010).

16. González-Rosa, J.M., Mercader, N. Cryoinjury as a myocardial infarction model for the study of cardiac regeneration in the zebrafish. Nature Protocols. 7, 782-788 (2012).

17. Chablais, F., Jaźwińska, A. Induction of myocardial infarction in adult zebrafish using cryoinjury. Journal of Visualized Experiments. 62, e3666 (2012).

18. Lauridsen, H., Pedersen, M. Rebuilding a heart: complete regeneration after myocardial infarction in the axolotl. The Federation of American Societies for Experimental Biology Journal. 28 (s1) (2014).

19. McCusker, C., Gardiner, D.M. The axolotl model for regeneration and aging research: a mini-review. Gerontology. 57, 565-71 (2011).

20. Dittrich, A., Thygesen, M.M., Lauridsen, H. 2D and 3D Echocardiography in the Axolotl (Ambystoma Mexicanum) Journal of Visualized Experiments. 141, e57089 (2018).

21. Mühlfeld, C., Nyengaard, J. R., Mayhew, T. M. A review of state-of-the-art stereology for better quantitative 3D morphology in cardiac research. Cardiovascular Pathology. 19, 65-82 (2010).

22. Thygesen, M.M., Rasmussen, M.M., Madsen, J.G., Pedersen, M., Lauridsen, H. Propofol (2,6diisopropylphenol) is an applicable immersion anesthetic in the axolotl with potential uses in hemodynamic and neurophysiological experiments. Regeneration (Oxf.). 4, 124-131 (2017).

23. Malvin, G. M., Heisler, N. Blood flow patterns in the salamander, Ambystoma tigrinum, before, during and after metamorphosis. Journal of Experimental Biology. 137, 53-74 (1988).

24. Juul-Belling, H., Hofmeister, W., Andersen, D.C. A systematic exposition of methods used for quantification of heart regeneration after apex resection in zebrafish. Cells. 9, 548 (2020). 